\title{
Éditorial: L'Introduction à la section spéciale sur la prévention des chutes
}

L'impulsion de cette section spéciale est née du désir de gérontologues d'avoir un plus grand engagement avec la question des chutes à la fin de la vie. Mon expérience au Royaume-Uni est que les chutes sont une grande préoccupation de la profession medicale, mais que cette préoccupation n'est pas tant partagée par les chercheurs en sciences sociales. Le British Geriatrics Society est ouvert à toutes les disciplines, même si, comme une branche plus définie de la médicine, il est naturellement composé principalement de gériatres et de professionales paramédicaux, commes les kinésithérapeutes. Les membres de la Société britannique de la gériatrie prennent la question des chutes au sérieux et ont un groupe d'intérêt special qui héberge une conférence internationale annuelle. La Société britannique de la gériatrie est également ouverte à toutes les disciplines, mais elle est plus multidisciplinaire, représentant le plus large éventail de disciplines des sciences sociales, des arts et des humanités. Contrairement aux gériatres, peu de membres de la Société britannique de la gériatrie mènent des recherches dans le domaine des chutes.

En tant que psychologue, je perçois bien la contribution qui peut être fait à la base de connaissances sur la prévention des chutes par les gérontologues et les spécialistes des sciences sociales. On comprend que les gériatres et les professionnels de la santé puissent s'intéresser particulièrement au problème des chutes parce qu'il y font face dans les soins de routine qu'ils prodiguent à leurs patients. Cependant, les psychologues de la santé, sociologues médicaux, et des collègues d'autres disciplines liées ont depuis longtemps pris un intérêt dans la santé et les soins des personnes âgées-alors, pourquoi ce manque d'intérêt dans les chutes ?

J'ai fait l'hypothèse qu'au moins l'une des raisons pour lesquelles les gérontologues britanniques ne sont pas encore pleinement engagés dans la recherche sur la prévention des chutes est peutêtre qu'ils connaissent mal le sujet. Pour contribuer à mettre les collègues au courant des développements actuels et des enjeux de la recherche sur la prévention des chutes, en 2009, j'ai présidé un colloque à la $38^{\mathrm{e}}$ conférence annuelle de la Société britannique de gérontologie à l'Université de Bristol, en Angleterre, intitulé : "L'État de l'art : La prévention des chutes chez les personnes âgées. » Les participants à ce symposium ont accepté d'écrire leurs articles pour publication, ainsi j'ai coordonné cette section spéciale et a invité des contributions supplémentaires des collègues connus pour leur expertise dans ce domaine.

\section{La Logique de la section spéciale}

Les chutes sont reconnues mondialement comme une menace majeure pour la santé et le bien-être des personne âgées, et sont associées à la morbidité, l'admission à l'hôpital et à la maison de soins infirmiers, et la mortalité. Les données statistiques sur la prévalence et la gravité des chutes sont présentées dans notre premier article de cette section spéciale, qui indique la gravité de la question des chutes plus tard dans la vie et sa préoccupation croissante, étant donné le vieillissement de la population. Bien qu'il existent d'excellentes sources d'informations déjà disponibles dans la littérature, cette section spéciale sera particulièrement utile aux lecteurs pour ses approches multidisciplinaires et actuelles. Le but de cette section spéciale est double. Premièrement, l'objectif est d'introduire le thème de chutes aux lecteurs auxquels ce domaine de recherche est mal connu, et, en ce faisant, d'expliquer les concepts d'une manière lisible, qui ne sont pas nécessairement accessibles à cause de la nature multidisciplinaire du domaine. Deuxièmement, l'objectif est d'amener les lecteurs au courant avec des commentaires sur l'état actuel du terrain et de présenter de nouvelles données dans le domaine sousdéveloppé des influences culturelles sur les idées reçues sur les chutes et leur prévention.

Cette section spéciale sur la prévention des chutes se distingue de deux manières. Tout d'abord, les contributeurs ont été choisis délibérément pour former une équipe multidisciplinaire ayant une expertise en la santé de la population, l'épidémiologie, la gériatrie, la psychologie et les sciences infirmières. En particulier, la domaine sous-représenté des facteurs psychosociaux est représenté dans deux articles. Deuxièmement, et cela est lié au premier point, ces manuscrits couvrent un large éventail de sujets pertinents pour l'étude et la pratique de la réduction des chutes. Chaque article est nouveau et, pris ensemble, ils couvrent les questions épidémiologiques, historiques et psychosociaux, ainsi que la preuve de la façon dont les chutes peuvent être évitées par les médecins.

\section{Le Contenu de la section spéciale}

La section spéciale contient cinq articles, dont les quatre premiers révisent la littérature sur les données probantes actuelles, tandis que l'article final présente de nouvelles données empiriques. Peel (Australie) 
commence la section en soulevant la question majeure de la santé publique des chutes, en raison de leur haute fréquence et leur gravité parmi les personnes âgées. La plupart des articles de recherche dans ce domaine citent des statistiques épidémiologiques brièvement, mais cette étude fournit une analyse approfondie de ces données. Peel note que la charge de la santé publique des chutes est appelé à augmenter, compte tenu d'une population mondiale vieillissante, et les risques accrus de chutes et de blessures liées aux chutes chez les plus vieux parmi les personnes âgées et ceux qui résident dans les établissements de soins. Peel souligne également l'augmentation du risque de chutes chez les sousgroupes de la population âgée, y compris les femmes et les personnes de faible statut socio-économique. Le deuxième article par Speechley (Canada) offre un examen unique de la littérature en présentant une perspective historique sur la façon dont le domaine s'est developpé avec une mise au point particulière à l'importance de la méthodologie. Cet examen port sur les principales études publiées dans ce domaine et, avec un accent sur la sophistication croissante des méthodes, explique les incohérences et les résultats contradictoires dans la littérature. Ensuite, Speechley racommande résolument un modèle plus simple d'expliquer l'équilibre physiologique et l'instabilité, plutôt que d'élaborer une liste exhaustive des facteurs de risque multifactoriel des chutes. Le troisième article de Martin (Royaume-Uni) explique comment les chutes doivent être comprise comme un syndrome gériatrique qui est étroitement lié aux étourdissements et aux syncopes. Un certain nombre de conditions médicales sont indiquées comme facteurs de risque de chutes et de fractures de fragilité, y compris la syncope, la démarche alterée, des troubles cognitifs, l'incontinence, des maladies chroniques, l'utilisation des médicaments, et l'ostéoporose. Martin présente des données pour l'évaluation clinique et le traitement sur mesure de ces facteurs de risque de chutes, mettant l'accent sur la compréhension du contexte dans lequel l'individu est tombé, afin de prévenir les chutes secondaires.

Le quatrième article par Nyman (Royaume-Uni) fournit pour la première fois un aperçu des facteurs psychosociaux qui influent sur l'engagement des personnes âgées à pratiquer des activités physiques pour la prévention des chutes. Il utilise la théorie du comportement planifié d'intégrer la recherche sur le rôle des différents facteurs influents, y compris les connaissances, les attitudes, la confiance, l'identité, et le contexte social. Cette recherche est importante, car il est régulièrement constaté que les conseils fondés et les interventions ne sont pas toujours prises par les personnes âgées, ni soutenue à long terme.

Ces articles de synthèse jettent les fondements pour le cinquième et dernier article par Horton et Dickinson (Royaume-Uni). Ils rapportent les données empiriques et qualitatives d'une étude des personnes âgées chinoises pour mettre en évidence le rôle sous-étudié de la culture et de la diversité dans les idées reçues sur les chutes et leur prévention. Leur étude indique que les personnes âgées chinoises peuvent être réticentes à aborder le sujet des chutes, de peur causer des inquiétudes à leurs enfants adultes. Elles peuvent ne s'engager que dans des activités culturellement familières, et peuvent avoir un sens du devoir moral de rester en bonne santé, mais elles estiment que les chutes qui se produisent sont le résultat de la malchance. Horton et Dickinson illustrent ces points et soulignent leur capacité à inhiber la participation des personnes âgées chinoises dans les interventions visant à prévenir les chutes, en plus de barrières de langue et d'alphabétisation.

De ces cinq articles, les lecteurs pourront mieux comprendre le problème très répandu de chutes ; comment a évolué la recherche sur les chutes; comment les chutes peuvent être évitées; et les facteurs psychosociaux et culturels qui influencent la participation des personnes âgées dans les interventions pour prévenir les chutes. J'espère que cette section spéciale aidera à atteindre l'objectif d'attirer davantage l'attention sur la question de chutes et, en particulier, encouragera un plus grand engagement par les gérontologues et les spécialistes des sciences sociales dans les recherches futures.

Samuel R. Nyman, Ph.D.

Redacteur invité 